Research Article

\title{
Towards Designing Personal Health Information Management System Based on Java
}

\author{
Yitao Chen ${ }^{1}$ and Linhua Wan $\mathbb{D}^{2}$ \\ ${ }^{1}$ The Third People's Hospital of Guizhou Province, Guiyang 550081, China \\ ${ }^{2}$ Hangzhou Normal University, Hangzhou 311121, China \\ Correspondence should be addressed to Linhua Wan; wlh86@zcmu.edu.cn
}

Received 17 October 2021; Accepted 11 November 2021; Published 26 November 2021

Academic Editor: Ateeq Rehman

Copyright (c) 2021 Yitao Chen and Linhua Wan. This is an open access article distributed under the Creative Commons Attribution License, which permits unrestricted use, distribution, and reproduction in any medium, provided the original work is properly cited.

\begin{abstract}
Health information management systems help gather, compile, and analyze health data to help manage population health and reduce healthcare costs. It can support the clinical decision, help diagnose individual patients, and improve patient care. In this study, a personal health information management system based on Java is presented. Based on the Java platform, the overall hierarchical structure of the system is designed including a health information management module, personal health data synchronization module, and full-text retrieval module, to realize the functions of the system. To improve the security of personal health information, the data encryption standard (DES) algorithm is implemented to encrypt and protect personal healthcare information. The system is evaluated in terms of health information acquisition accuracy, information security, and system response time. To verify the robustness of the proposed health information management system, it is compared with two related studies. The maximum health information acquisition accuracy of the system is $99 \%$, the safety factor reported is above 0.9 , and the maximum response time is only $0.8 \mathrm{~s}$. The experimental results show that the designed personal health information management system can collect health information more accurately and ensure the security of information, and the overall response time is shorter.
\end{abstract}

\section{Introduction}

A big challenge that patients face today in healthcare is the unavailability of access to their health information. Idyllically, patients will have a complete and updated version of their health information, which can be made accessible to any provider that they wish to use it. As patients move, travel, and get their care from different care providers, their treatment records are shared across multiple sites where they have received treatment. These medical records are usually not shared and updated unless the sites are part of a network of providers that share common medical information systems [1]. Past treatment records are an essential input in clinical decisionmaking, and without the consolidation of all related medical information, there is a risk of inefficient treatment, delays in diagnosis, and medical errors.
Recently, people's living standards have significantly improved. At the same time, the concept of healthy life has attracted more and more attention. People's attention to personal healthcare and disease prevention has become stronger and stronger. At the same time, the accelerating pace of life, food safety, and the increasing trend of population aging have caused a strong impact on traditional medical care and healthcare methods. How to conveniently realize and comprehensively master their health information, realize the prevention of chronic diseases and more accurate diagnosis, and realize personal health information management has become a topic of increasing concern [2].

The rapid development of information communication and computing power [3] provides great potential for medical information access, collection, sharing, and analysis. These technologies provide exceptional chances to support 
next-generation healthcare systems. A major fence today is how to personalize healthcare services and to help people to enhance current offered healthcare services and applications. Zhe and Yongli [4] proposed a healthcare data management system based on integrated learning. The system is comprised of multiple layers in which the administrator operates and manages the operation layer to achieve system control, regulation, and application. The big data analysis layer is used to obtain the health data prediction results fused with multidimensional parameter information. The bagging-integrated learning method is combined with the ELM predictive model to obtain a high-precision strong learning model to achieve effective interpretation of different multidimensional all-cloud health big data. Li and Dongxin [5] proposed a health data management system based on the $\mathrm{B} / \mathrm{S}$ architecture. A comprehensive student mental health database has been constructed in this system, and a mental health diagnosis and treatment plan for college and universities student has been established for remote consultation, self-diagnosis, on-site evaluation, and psychotherapy. $\mathrm{Hu}$ et al. [6] developed a healthcare information management system based on virtual reality. Based on graphics and image processing techniques, the management mode is presented in $3 \mathrm{D}$ visualization, which makes the management of the data center more intuitive, and managers can be more proactive and convenient to manage. The authors in [7] discussed the structure of the Java EE platform and analyzed the Strust2, Spring, and Hibernate frameworks. In this system, the solution of the remote health information service system is presented through the integration of these three frameworks. The system was effective in providing a method for online diagnosis and health consultation. A distributed health information system that uses the service-oriented architecture as a basis for developing, implementing, installing, managing, and calling e-healthcare services was presented in [8]. The system is based on multimedia input and output, such as graphics and speech, which makes the system more pretty to users who are not computer-oriented. Li et al. [9] developed an intervention of subhealth management information system using a lightweight Java EE framework based on Struts, Hibernate, and Spring. The system is divided into three layers, web layer, service layer, and PO layer. The system can provide better maintainability and reliability in healthcare services. Ngai et al. [10] proposed a radio frequency identification-based healthcare information management system for the healthcare industries. They investigated the application of radio frequency identification technology for building the healthcare information management system for providing effective services in transforming and optimizing healthcare practices in the industry. Olamide et al. [11] constructed a hospital management system that provides improved administration and control, more patient care, and improved profitability. The system is based on Java as the front-end software and has connectivity with the back-end software.

To realize the security management of personal health information, a personal health information management system based on Java is presented in this study. With the support of the Java platform, the reliability of the personal health information management system is improved, and the encryption algorithm is used to encrypt and securely transmit personal health information.

The rest of the paper is ordered as follows. In Section 2, the proposed healthcare information management system is presented and the different modules and functions are illustrated. In Section 3, the results of the proposed system are discussed, and the conclusion is given in Section 4 .

\section{Personal Health Information Management System Based on Java}

Java platform is a specification and standard for developing distributed enterprise applications. It provides developers with a set of powerful application programming interfaces (APIs) to facilitate users' network application development. Developers can build reusable and flexible web applications through this platform. At present, the Java platform has become one of the most widely used web application development technologies in global enterprise application information systems [12]. Java uses a distributed multilayer application model, divides the application logic into different components according to functions, and divides the two-layer model into four layers, and the layers are independent of each other. Each layer is assigned a specific service. The idea of layering enables the client to solve the problems of data reception and data display. The overall structure of the proposed personal health information management system constructed is shown in Figure 1.

The disruption of the different layers in the layered architecture of Java is given as follows:

(i) Client Layer. This layer directly interacts with system users. The client layer presents the result data information of the server background processing to users. The Java platform supports multiple types of users, such as hypertext markup language (HTML) and Java Applet, usually a browser.

(ii) Presentation Layer. This layer is mainly managed by the web container and used to receive user requests. The presentation layer passes the received data to the business logic layer and returns the results processed by the business logic layer to the client layer. Usually, the function of the presentation layer is realized by Java server pages (JSP) or Servlets [10].

(iii) Business Logic Layer. It provides services for the presentation layer to process business logic and provides necessary interfaces. The business logic layer stores the data provided by the presentation layer into the information system layer after corresponding processing and also returns the processing results to the presentation layer.

(iv) Information System Layer. The services of this layer are mainly implemented in the database system for storing and retrieving information.

Since Java is a platform-independent language, products based on the Java platform can run on any operating system and hardware in the market. Therefore, products developed 


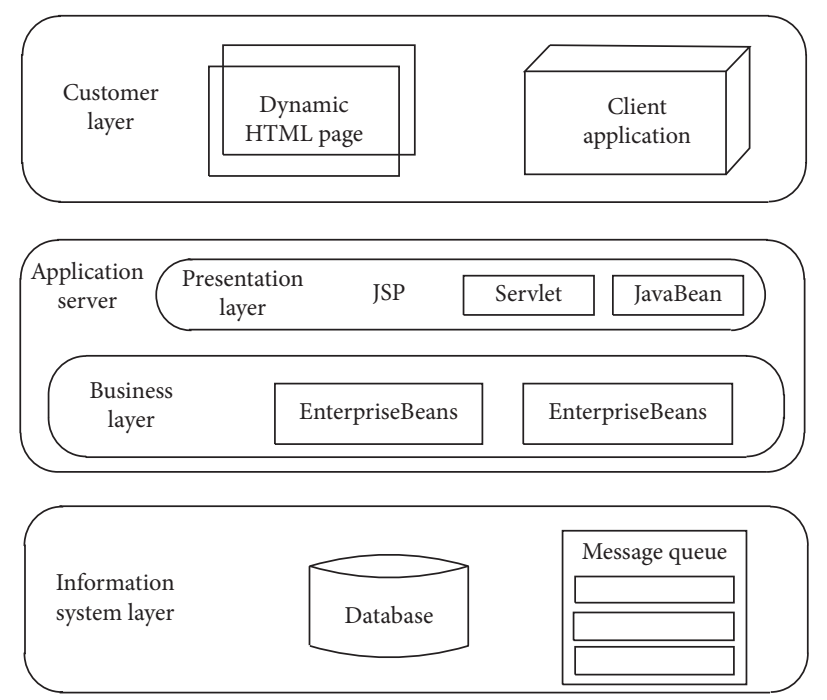

Figure 1: Structure of the personal health information management system.

on the Java platform can reduce secondary development caused by platform upgrades and project migration. The Java platform provides an excellent platform for software application developers to develop efficient, flexible, and easyto-use web applications [13].

\subsection{System Hardware Design}

2.1.1. Health Information Management Module. A personal health information system should be easy and convenient to work because its users are mostly patients and clinicians. The users should be able to use the client via a web browser as the operation of the system interface, and they can be able to use the web interface directly [14]. Based on the above consideration, this system is developed and designed based on the Java EE platform. The architecture of the personal health information management module is depicted in Figure 2.

Personal file management is mainly divided into two file management categories: visit report and health file. The visit report manages the user's medical information records in the form of file management, such as hospital information, department information, medical personnel information, and inspection report. The personal health information management system also maintains the most basic and perfect health file information for individual users. Health files should meet national standards, have comprehensive information coverage, and realize the characteristic management of the whole life [15].

The health data management function mainly realizes the classified management of personal sign data, cardiovascular data, and other health data. As the information data with obvious personal health characteristics, personal sign data, and cardiovascular data have the characteristics of relatively mature data measurement technology, relatively stable data changes, and serious impact of abnormal data on physical health. They are widely used in health examination and the prevention of chronic diseases.
In addition to core functions such as health information management, the health information management module also provides health service management.

(1) Healthcare Knowledge Popularization. For common health problems, the information management module can recommend medical and health knowledge popularization for users, improve users' medical and health knowledge level, and require the content of medical and health knowledge base to be simple, easy to understand, rigorous, and reliable.

(2) Visit Information Recommendation. This module comprehensively recommends the medical institution information with user characteristics according to the existing user's health information, the basic information of the connected medical institution, the specialty of the department, and the comprehensive medical ability. Moreover, it can also provide users with geographic location information of medical institutions.

(3) Personal Health Information Sharing. This module can be used by patients to query their health files, medical reports, and health data by authorizing other users [16].

(4) Complaints and Suggestions. In addition to providing users with the input and suggestion function of the personal health information management system platform, users can also be provided with the ability to evaluate and complain about medical institutions and medical personnel. Users' evaluation and complaint suggestions can be used as an assessment index of the comprehensive ability of medical institutions and medical personnel [17].

2.1.2. Personal Health Data Synchronization Module. It is essential to analyze the synchronization of patient data among health facilities using electronic medical records systems. The application of modern technology in the healthcare system 
aims to upsurge the reliability, convenience, and productivity of delivered healthcare services [18]. The proposed personal health information management system is an application system based on a medical system database. In the development of such systems, it is indispensable to establish synchronization mechanisms and data sharing between the different databases. In this study, encryption techniques are used to encrypt data transmission. Moreover, firewalls and other security measures are also added for protection. The network topology of synchronous transmission of the personal health data is shown in Figure 3.

To synchronize data between the webserver service center and the data center of the platform, a general transmission protocol must be designed, and data transmission can be carried out based on this transmission protocol. Because the data center needs to synchronize data with multiple application systems, the coupling of the system must also be considered when designing the synchronization network topology; that is, in the design process, the data dependence between systems should be reduced as much as possible.

2.1.3. Full-Text Retrieval Module. To achieve a comprehensive retrieval of personal health and medical data, the full-text retrieval module is designed using the integrated model of Solr and HBase. Solr is an enterprise-level search server development framework based on Apache Lucene. It uses an API interface similar to RESTful Web Service. Therefore, its indexing method is similar to the Key-Value pair used by HBase. It is very convenient to call, which makes the integrated development based on Solr and HBase relatively convenient. The overall structure of the full-text search module is shown in Figure 4.

2.2. System Software Design. Privacy-preserving is at present a highly active domain of the healthcare system. Ensure safe and reliable medical data are stored. It is very suitable for software and hardware requirements as encryption and decryption take the same algorithm. In this algorithm, only the encryption function needs to be reversed and the round keys to be taken in opposite order [19]. To protect users' health data, data encryption standard (DES) symmetric encryption is used to encrypt personal health data. The main process of DES symmetric encryption is to perform calculations fixedly. There are 64 bit plain texts, which are converted into 56 bit secret keys to achieve encryption. The schematic diagram of DES symmetric encryption is shown in Figure 5.

Each round of calculation is a set of procedures for DES symmetric encryption. The single set of calculation procedures is shown in Figure 6.

The DES symmetric encryption first defines the key by mathematical logic, which is defined as follows:

$$
K=\lim _{0 \longrightarrow \infty} M \bigcap_{i=1}^{n} X_{i} g !
$$

where $M$ represents the plaintext of personal health privacy information, $X_{i}$ is the bandwidth of the transmission network, and $g$ shows the local area network environment for personal health information transmission.

Based on the definition of secret key space $K$, the ciphertext encryption method is defined, which is computed using the following equation:

$$
Q=\frac{W_{0}\left(\partial^{2} q_{k} \mathrm{~d} x\right)}{K}
$$

In equation (2), $K$ represents the length of the secret key space, $q_{k}$ represents the safety factor, and $W_{0}$ represents the amount of plaintext data in personal health data.

Using the ciphertext encryption method, the plain text is encrypted and calculated. The computation process is given as

$$
C=\frac{Q}{S}+Z\left(\partial^{2} a q_{k} \mathrm{~d} x\right)
$$

where $S$ is the encryption rule variable and $Z$ represents the encryption environment coefficient, and its value range is $[0$, $1]$. Generally, it takes the upper limit of 0.65 , and $a$ represents the maximum content that the equipment can run. Using equation (3), the encryption processing of personal health data is completed. The encryption data flow process is shown in Figure 7.

\section{Experimental Verification}

In this study, a personal health information management system based on Java is developed and implemented. To verify the performance of the system, the system performance test experiment is carried out. The test environment configuration information of the system is shown in Table 1.

The system login interface is shown in Figure 8.

After the system logs in successfully, the system performance verification experiment is started. The overall experimental scheme is set as follows: taking the health information acquisition accuracy, information security, and system response time as the experimental comparison indicators, the proposed personal health information system is verified and compared with the integrated learning system proposed in reference [4] and with the B/S architecture system proposed in reference [5].

3.1. Health Information Collection Accuracy. For scientific instruments, it is essential to be both accurate and precise. This is important because bad equipment, poor data processing, or human error can lead to inaccurate results that are not very close to the truth. The accuracy represents how precisely the measured values reflect the real or the original values of the personal health information system [20]. Figure 9 shows the comparative results of the proposed health information system with the personal health information systems presented in $[4,5]$, respectively.

It can be seen that in a long experimental time, the information acquisition accuracy of the proposed health information system always maintains a high level, and the maximum acquisition accuracy reaches $99 \%$. The acquisition accuracy of the system based on integrated learning and the 


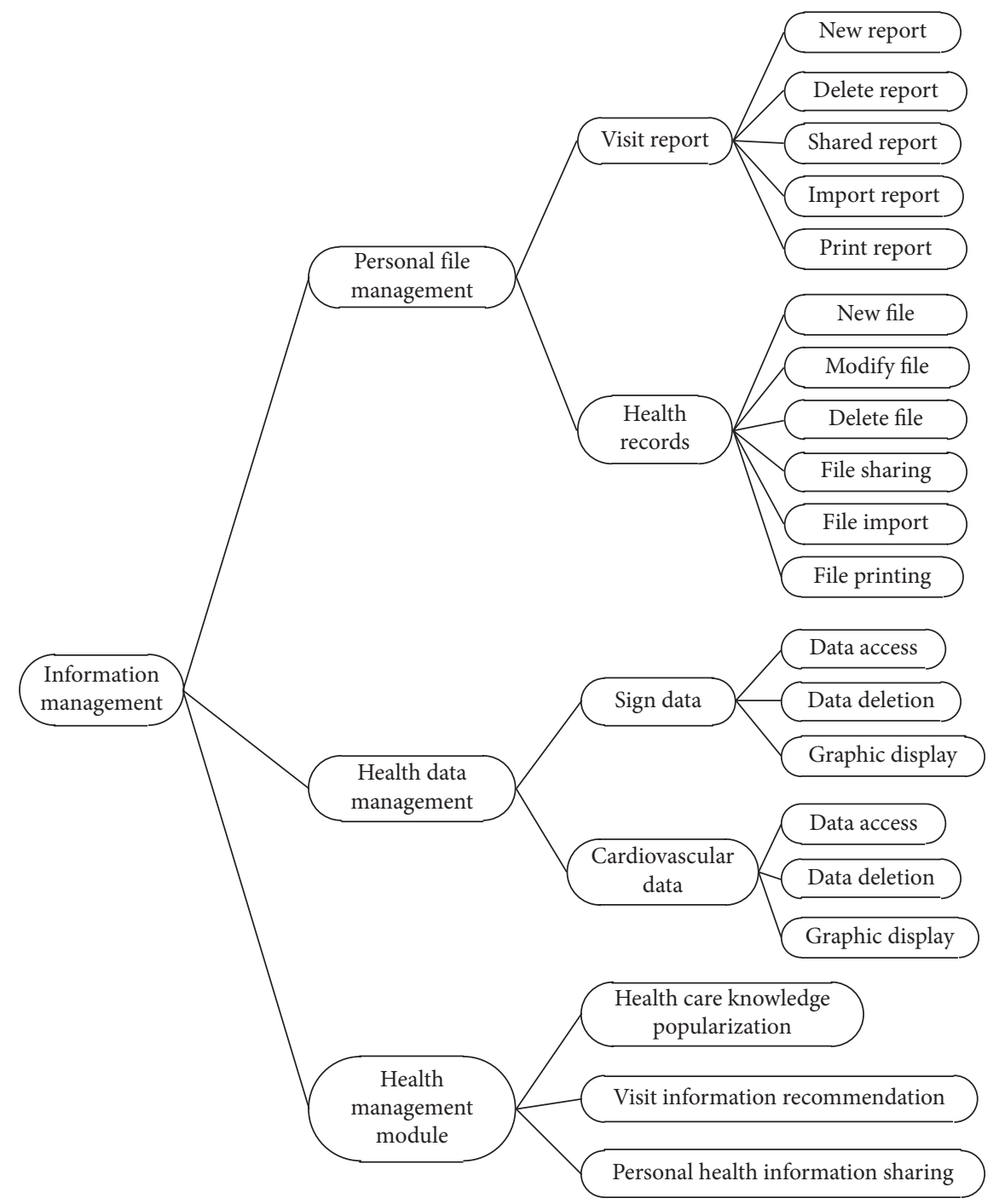

FIgURE 2: Functional structure of health information management module.

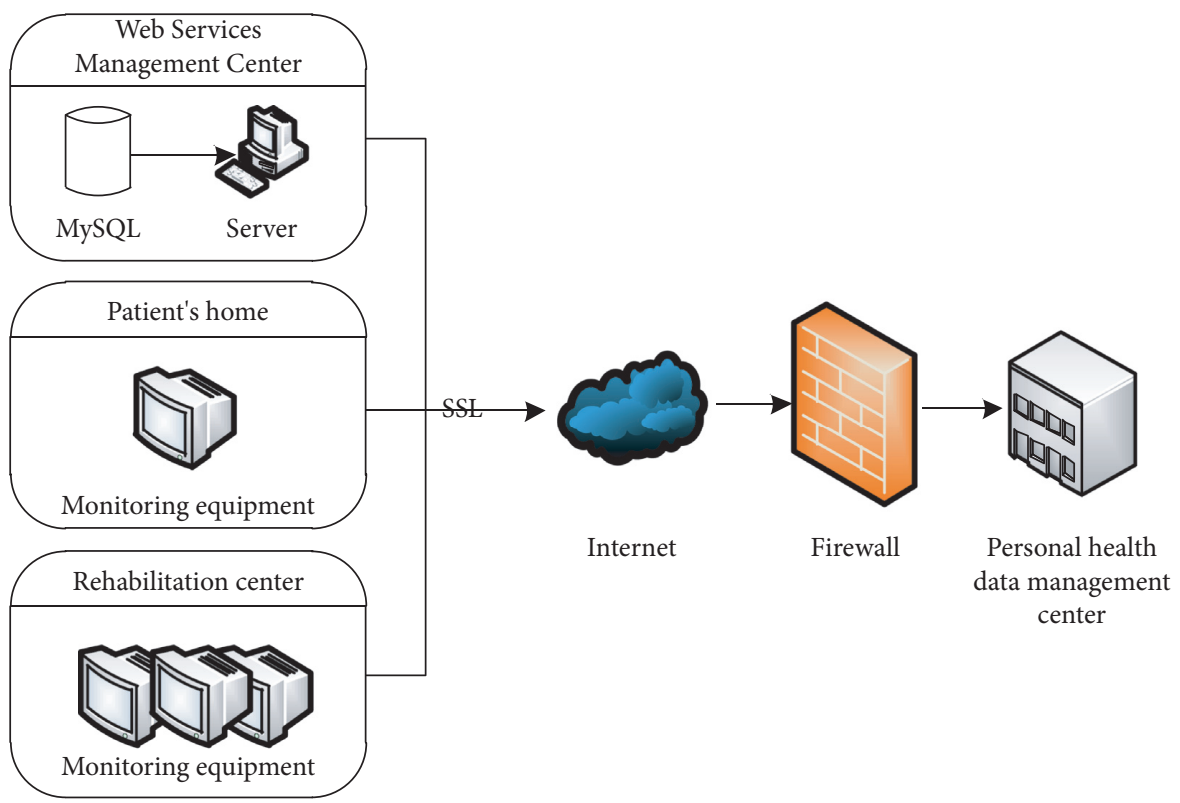

Figure 3: Network topology of synchronous transmission of personal health data. 


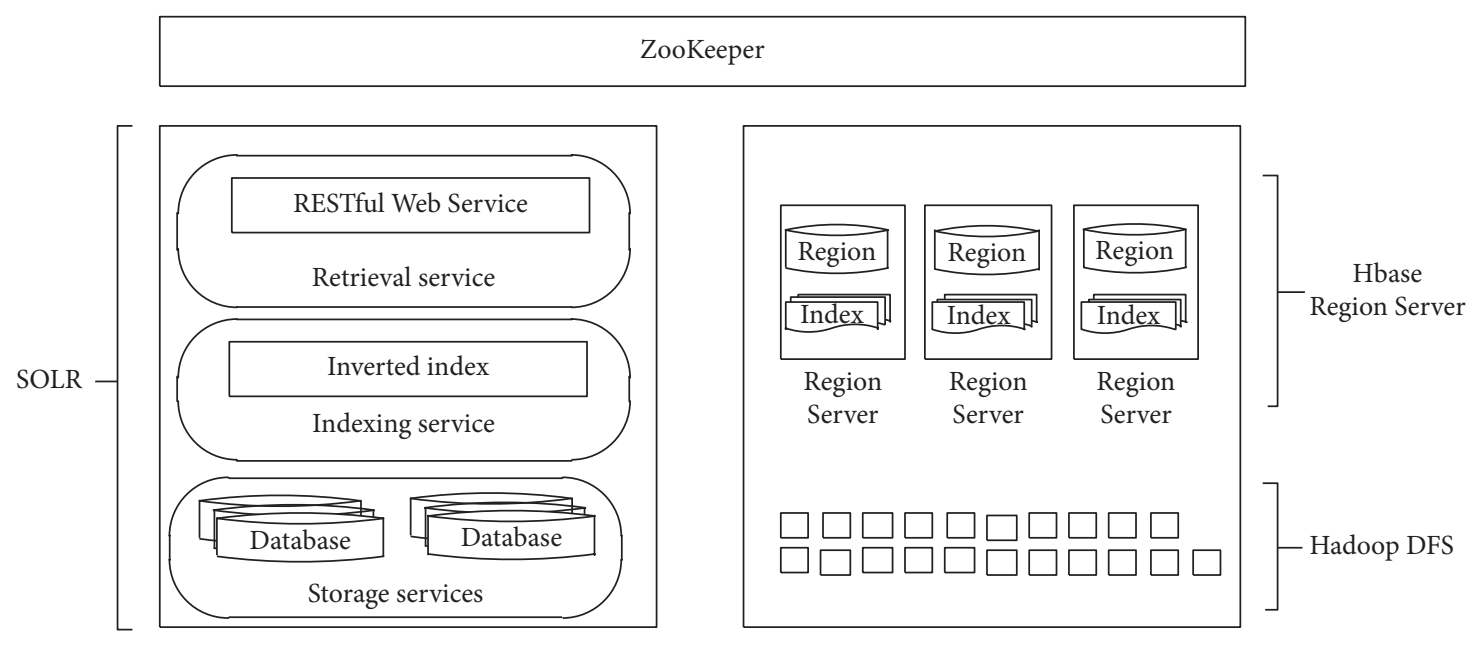

Figure 4: The overall structure of the full-text retrieval module.

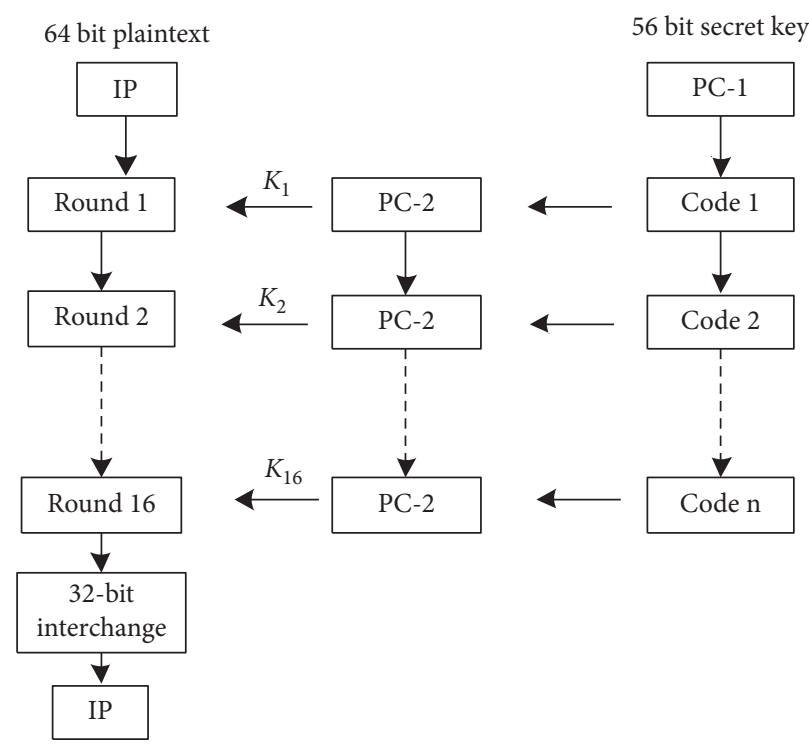

FIgURE 5: Schematic diagram of DES symmetric encryption.

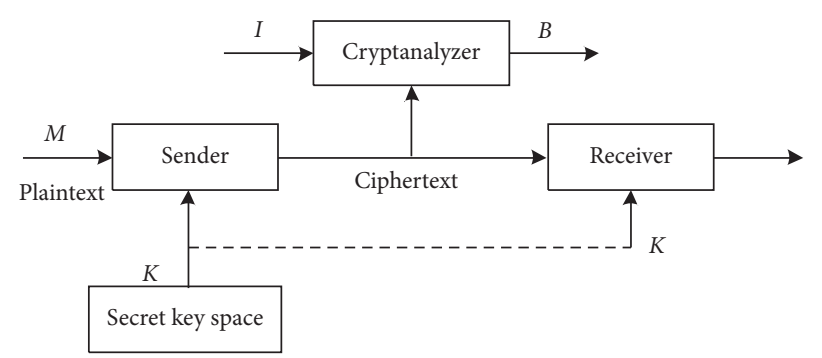

FIgURE 6: Single-group DES symmetric encryption process.

system based on B/S architecture fluctuates greatly, and the maximum acquisition accuracy of the two systems is always less than $90 \%$. This shows that the proposed health information system is reliable, and its performance is stable under different experimental conditions.
3.2. Information Security. Information security is the protection of information and information systems from unauthorized access, use, disclosure, disruption, modification, or destruction. Information security is achieved by ensuring the confidentiality, integrity, and availability of 


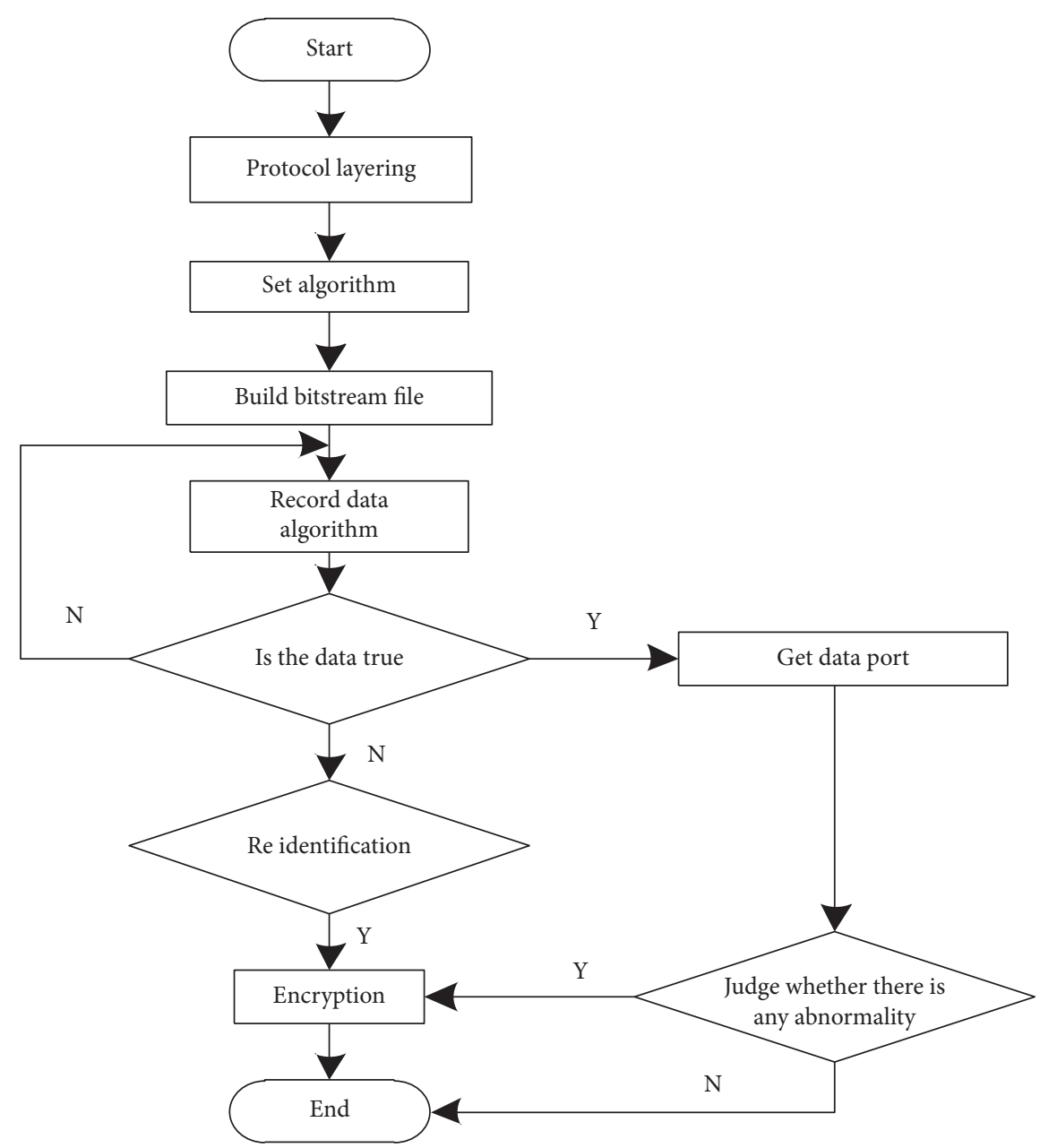

Figure 7: Encryption flow chart.

TABLE 1: Test environment configuration information.

\begin{tabular}{lcc}
\hline Client side & Configuration & Parameter \\
\hline & CPU & MediaTek MT6589T, quad-core, 1.5 GHz \\
Mobile terminal & Memory & 1 GB RAM + 4G ROM \\
& System & Android 4.2.1 \\
\hline \multirow{3}{*}{ Computer terminal } & Bluetooth & Bluetooth 4.0 \\
& CPU & Intel core i5 4440 \\
& Memory & 4 GB \\
\hline
\end{tabular}

information. Because of personal health information and secret information of users, the system needs to protect users' health information and avoid illegal and unauthorized access. Therefore, a personal health information management system is required to have high information security. The information security factor is a parameter between 0 and 1 . The closer it is to 1 , the higher the security of information is. The information security comparison results of the three systems are shown in Table 2. It can be seen that the safety factor of the proposed personal health information is always maintained above 0.9 , while the highest safety factor of the two comparison methods is only 84 . The average safety factor of the proposed system is 0.968 , the average safety factor of the system based on integrated learning is 0.695 , and the average safety factor of the system based on B/S architecture is 0.692 , respectively. Therefore, it is verified that the proposed system can improve the security of personal health information.

3.3. System Response Time. System response time is the time between when the user initiates an action and when the health information system starts to display the result. 


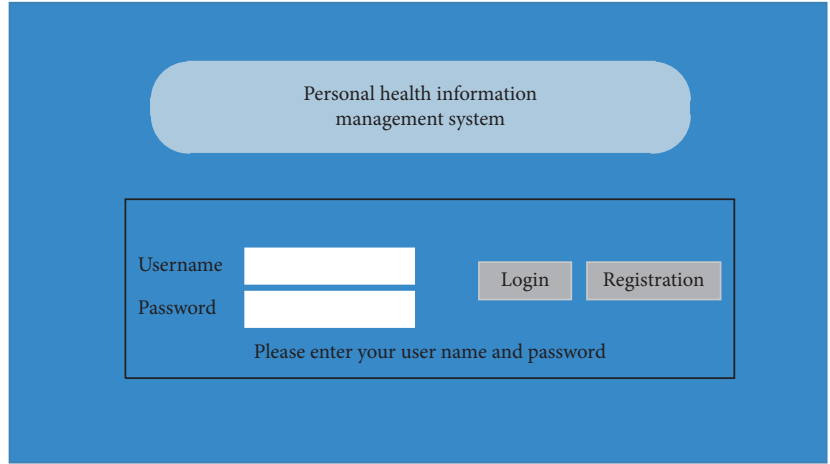

Figure 8: System login interface.

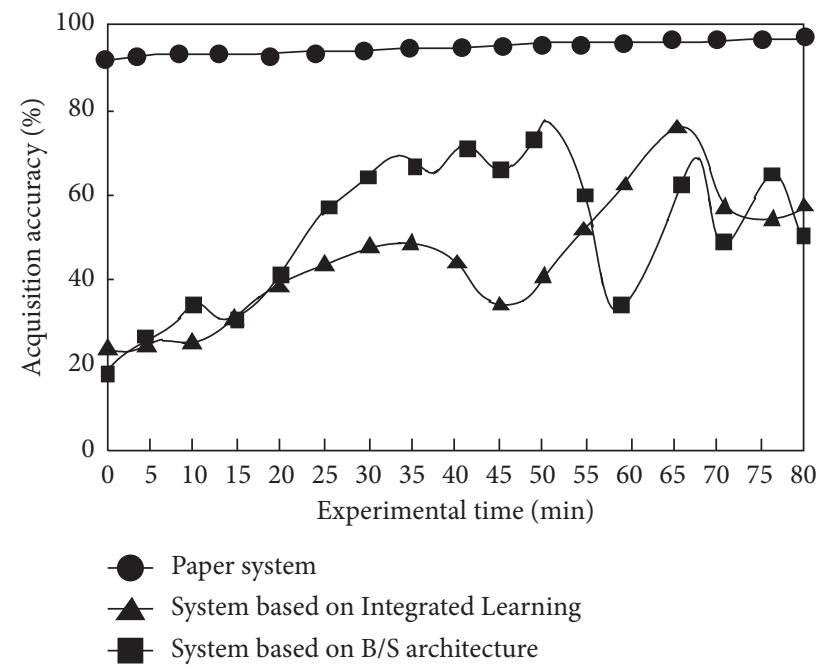

FIgURE 9: Comparison results of health information acquisition accuracy.

TABLE 2: Comparison results of information security.

\begin{tabular}{|c|c|c|c|}
\hline \multirow{2}{*}{ No. of experiments/time } & \multicolumn{3}{|c|}{ Information security factor } \\
\hline & Proposed system & System based on integrated learning & System based on B/S architecture \\
\hline 1 & 0.98 & 0.76 & 0.68 \\
\hline 2 & 0.96 & 0.72 & 0.84 \\
\hline 3 & 0.97 & 0.69 & 0.82 \\
\hline 4 & 0.97 & 0.81 & 0.78 \\
\hline 5 & 0.98 & 0.74 & 0.77 \\
\hline 6 & 0.95 & 0.65 & 0.62 \\
\hline 7 & 0.96 & 0.63 & 0.58 \\
\hline 8 & 0.95 & 0.63 & 0.59 \\
\hline 9 & 0.99 & 0.61 & 0.64 \\
\hline 10 & 0.97 & 0.71 & 0.60 \\
\hline Mean value & 0.968 & 0.695 & 0.692 \\
\hline
\end{tabular}

Health information systems must answer to external interactions in the environment within a predetermined amount of time. Health information systems must provide the correct result and generate it in a timely manner. This means that response time is as important as producing correct results. Real-time systems must be developed to meet these response times. Hardware and software must be developed to support response time requirements for these systems. The response time comparison results of the three systems are shown in Table 3. 
TABLE 3: Comparison results of system response time.

\begin{tabular}{|c|c|c|c|}
\hline \multirow{2}{*}{ No. of experiments/time } & \multicolumn{3}{|c|}{ System response time (s) } \\
\hline & Proposed system & System based on integrated learning & System based on B/S architecture \\
\hline 1 & 0.5 & 6.4 & 5.4 \\
\hline 2 & 0.6 & 5.6 & 6.5 \\
\hline 3 & 0.5 & 5.9 & 6.8 \\
\hline 4 & 0.7 & 6.3 & 6.4 \\
\hline 5 & 0.8 & 6.9 & 6.2 \\
\hline 6 & 0.5 & 5.8 & 7.1 \\
\hline 7 & 0.6 & 5.9 & 6.6 \\
\hline 8 & 0.7 & 5.9 & 6.4 \\
\hline 9 & 0.6 & 6.5 & 6.7 \\
\hline 10 & 0.8 & 6.1 & 6.2 \\
\hline
\end{tabular}

Observing the comparison results of system response time, it can be found that the response time of the proposed system is much lower than that of the two existing health information systems. The maximum response time of the proposed system is $0.8 \mathrm{~s}$, that of the integrated learning system $6.1 \mathrm{~s}$, and that of the system based on B/S architecture is $7.1 \mathrm{~s}$, respectively. This proved that the system is much faster than the existing personal health information systems in providing feedback to the patients and clinicians.

\section{Conclusion}

In this paper, we presented a personal health information management system based on a Java that uses the serviceoriented architecture as a basis for designing, implementing, deploying, managing, and invoking e-healthcare services. We developed a web-based personal health information management system that can be used by patients to collect and manage their health information, request self-referrals, and store a record of their consultations. The system is composed of multiple modules, with each module providing different healthcare functions. To verify the robustness of the proposed health information management system, we compared it with two related studies, and the results were found better than the state-of-the-art models. The experimental results show that the designed personal health information management system can collect health information more accurately and ensure the security of information, and the overall response time is shorter. The maximum health information mining of this system is $99 \%$, the safety factor is always above 0.9 , and the maximum response time is only 0.8 . Therefore, it shows that the proposed personal health information management system can improve the overall management performance from many aspects.

\section{Data Availability}

The data used to support the findings of this study are available from the corresponding author upon request.

\section{Conflicts of Interest}

The authors declare that there are no conflicts of interest.

\section{References}

[1] S. Fang, "Simulation of health information symptoms in central Chinese clinical data," Computer Simulation, vol. 35, no. 2, pp. 350-353, 2018.

[2] H. Wang, N. Wang, M. Li, S. Mi, and Y. Shi, "Student physical health information management model under big data environment," Scientific Programming, vol. 2021, no. 1, 10 pages, Article ID 5795884, 2021.

[3] C. N. Umstead, K. M. Unertl, and N. Lorenzi, L. L. Novak, Enabling adoption and use of new health information technology during implementation: roles and strategies for internal and external support personnel," Journal of the American Medical Informatics Association, vol. 28, no. 7, pp. 1543-1547, 2021.

[4] Z. Zhe and T. Yongli, "Design of all-cloud health big data integration system based on integrated learning," Modern Electronics Technique, vol. 43, no. 22, pp. 181-188, 2020.

[5] H. Li and W. Dongxin, "Research and design of university psychological health information management system based on B/S," Electronic Design Engineering, vol. 26, no. 15, pp. 124-128, 2018.

[6] L. Hu, Z. Kemin, and Y. Hao, "Design of information management system based on virtual reality technology," Computer Technology and Development, vol. 30, no. 3, pp. 157-161, 2020.

[7] Y. Huo, Z. Cui, T. Liu, X. Zuo, and H. Wang, "Design of java EE-based remote health service system," in Proceedings of the IEEE, 2014 Sixth International Conference on Intelligent Human-Machine Systems and Cybernetics, pp. 339-342, Hangzhou, China, August 2014.

[8] F. Kart, G. Miao, L. E. Moser, and P. M. Melliar-Smith, "A distributed E-healthcare system based on the service oriented architecture," in Proceedings of the 2007 IEEE International Conference on Services Computing (SCC 2007), Salt Lake City, UT, USA, July 2007.

[9] Z. B. Li, J. Q. Du, H. Zhu, W. W. Xiong, and Z. Y. Liu, "An assessment and intervention of sub-health management information system based on lightweight java EE framework," Applied Mechanics and Materials, vol. 411-414, pp. 223-226, 2013.

[10] E. W. T. Ngai, J. K. L. Poon, F. F. C. Suk, and C. C. Ng, "Design of an RFID-based healthcare management system using an information system design theory," Information Systems Frontiers, vol. 11, no. 4, pp. 405-417, 2009.

[11] O. Olamide, E. Adedayo, and O. Abiodun, "Design and implementation of hospital management system using java," 
IOSR Journal of Mobile Computing \& Application, vol. 2, no. 1, pp. 32-36, 2015.

[12] O. Can and D. Yilmazer, "Improving privacy in health care with an ontology-based provenance management system," Expert Systems, vol. 37, no. 1, pp. 134-139, 2020.

[13] Y. Li, X. Yuan, and R. Che, "An investigation of task characteristics and users' evaluation of interaction design in different online health information systems," Information Processing \& Management, vol. 58, no. 3, pp. 104-108, 2021.

[14] A. Chrysantina, G. Sanjaya, M. Pinard, and N. M. Hanifah, "Improving health information management capacity with digital learning platform: the case of DHIS2 online academy," Procedia Computer Science, vol. 161, no. 1, pp. 195-203, 2019.

[15] A. V. Kumar, M. S. Sujith, and K. T. Sai, "Secure multiparty computation enabled E-healthcare system with homomorphic encryption," IOP Conference Series: Materials Science and Engineering, vol. 98, no. 12, pp. 22-27, 2019.

[16] S. E. Hajjami, M. Berrada, and M. Harti, "Using semantic web technologies and multi-agent system for multi-dimensional analysis of open health data," Journal of Information \& Knowledge Management, vol. 19, no. 3, pp. 205-211, 2020.

[17] C. Kawila, G. W, G. Otieno, and W. Mwaura-Tenambergen, "The role leadership style plays in the integration of health management information system," International Journal of Computer Applications, vol. 181, no. 2, pp. 1-5, 2018.

[18] L. Kwao, R. Millham, and E. Opanin, "An integrated success model for adopting biometric authentication technique for district health information management system 2, Ghana," International Journal of Computer Applications, vol. 177, no. 40 , pp. 1-16, 2020.

[19] S. Aithal, "Allied medical and health science and advanced telecommunications: emerging utilizations and its need in Indian healthcare system," Social Science Electronic Publishing, vol. 32, no. 18, pp. 193-199, 2018.

[20] T. Sinthamrongruk, K. Dahal, and J. Yanchinda, "Heuristic and fuzzy scheduling synergy with knowledge acquisition of resource selection system development for home healthcare service," International Journal of Agile Systems and Management, vol. 11, no. 3, pp. 247-252, 2018. 